\title{
USE OF CCD DETECTORS FOR SPECTROSCOPY AND SCATTERING EXPERIMENTS
}

\author{
Roy CLARKE \\ Department of Phvsics, The Unicersity of Michigan. Ann Arhor. MI 48109. USA
}

\begin{abstract}
The application of CCD detectors in X-ray synchrotron radiation experiments is described. Performance characteristics such as hıgh dynamıc range, versatıle readout and data storage modes, and time-resolved capabilitıes promise interestıng possibılities for future experiments on high-brillance sources The operation of CCDs as high-resolution position-sensitive detectors, used in conjunction with dispersive optics, is illustrated by recent results on near-edge reflectivity from multilayers and magnetic absorption experiments with circularly polarized X-rays.
\end{abstract}

\section{Introduction}

The construction of high-brilliance synchrotron rad1ation sources based on insertion devices will open up exciting new areas of X-ray and UV research in the coming decade. In order to exploit the unique characteristics of such facilities, especially the low emittance and the timing structure, it will be necessary to develop detector systems with capabilities far superior to those presently in use. With investment in new sources such as the Advanced Light Source (Lawrence Berkeley Laboratory), Advanced Photon Source (Argonne National Laboratory) and the European Synchrotron Radiation Facility (Grenoble) running currently in excess of $\$ 10^{9}$, there is certainly an urgent case for a vigorous $R \& D$ effort in detector technology.

The detectors envisioned for future synchrotronradiation applications will need to handle very large fluxes (i.e., have large dynamic range), and, for many experiments, fast readout will be required. This is particularly important for time-resolved studies, one of the major frontiers of synchrotron radiation research [1]. The latter requirements raise the additional question of how to manage the formidable amounts of data that are generated in such experiments. New detector development therefore requires attention to all aspects of the technology, from the front-end and readout electronics to data evaluation and processing.

A third component of any synchrotron radiation experiment, in addition to the source and the detector, is the beamline optics. By optımizing monochromators, mirrors and other optical elements to match the experimental situation and the particular detection scheme, new kinds of time-resolved experiments have been feasible on currently operating sources [2-4].

In this paper I will describe a developmental effort to evaluate the potential of some solid-state electronic detectors for synchrotron radiation applications. In an ongoing collaboration with Argonne National Laboratory, we have built and tested a prototype charge coupled device (CCD) detector which has many of the desirable characteristics one is lookıng for in a versatile detector system [5,6]. In particular we have demonstrated its usefulness as a high-resolution, two-dimensional, position-sensitive detector for spectroscopic and small-angle scattering studies. The performance of the CCD detector and its potential for novel types of synchrotron radiation experiments will be illustrated with examples of recent results on the dispersive beamline at LURE in Orsay, France.

\section{CCD detector and readout scheme}

The CCD used in the present detector is the Texas Instruments (TI) 4849 chip [7] consistıng of 584 rows of pixels arranged in 390 columns. Each pixel is $22.4 \mu \mathrm{m}$ square with a full-well capacity of approximately $2 \times 10^{5}$ electrons. The CCD chip is cooled thermoelectrically to $-50^{\circ} \mathrm{C}$ to reduce thermal generation of electron-hole pairs. Fig. 1 shows the CCD optically coupled to a 40-mm-diameter phosphor screen, the mode in which most of our experiments have been performed to date. The lens coupling shown here is relatively inefficient, producing roughly one optical photon at the CCD chip for every X-ray photon incident at the surface of the phosphor. This type of front-end has obvious limitations for flux-limited scattering experiments but can actually be advantageous [6] for adsorption spectroscopy in terms of signal/noise.

\section{Virtual phase design}

An interestıng feature of the so-called "virtual phase" CCD architecture [8] introduced by TI is its enhanced resistance to radiation damage [9]. This offers the possi- 


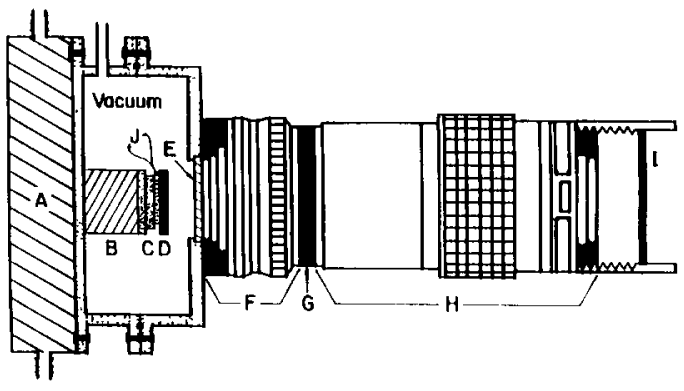

Fig. 1 The CCD detector with optical coupling to a phosphor screen. A. water cooled heat exchanger; B: copper base, C: thermoelectric cooler; D: CCD chip; E: quartz window; F. 50 mm f. 2 camera lens; G: Irss; H: $200 \mathrm{~mm}$ f 4 camera lens; I: phosphor screen (Trımax 2, P43) and beryllum window, J: thermocouple.

bility of operating the CCD under dırect illumination by X-rays; a relatively deep inversion layer $(\sim 12 \mu \mathrm{m})$ in this design is well matched to $\mathrm{X}$-rays in the energy range of 5-7 keV. Operated in this manner, without the phosphor screen/lens combination, each X-ray photon produces typically several hundred electron-hole pairs. Thus the detector gain is greatly enhanced at the expense of the dynamic range and, over the long term, accumulation of radiation damage.

The overall chip structure is shown in fig. 2. Charge produced by photons incident on the front side of the CCD is stored in potential wells created in the MOS-type inversion layer under each gate electrode. Readout of the chip is accomplished by transferring charge from pixel to pixel using a series of clocking pulses. In most CCDs a three-level gate structure is employed whereas the TI design uses a single phase in conjunction with ion-implanted "virtual electrodes" to create the asymmetric well structure. While radiation hardness is claimed to be an advantage of the virtual phase design, the deep ion implants can themselves lead to damage in

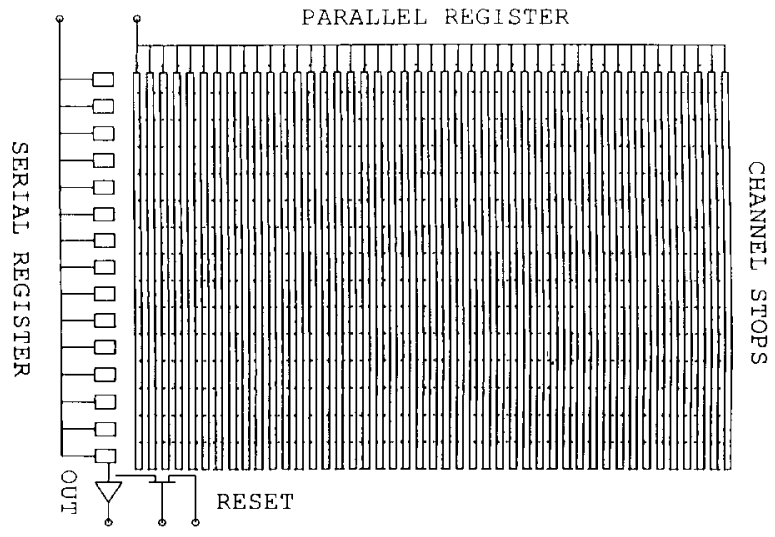

Fig. 2. Schematic view of the electrode structure of the virtualphase CCD chip the silicon lattice and subsequent degradation of the charge transfer efficiency. Accordingly, some manufacturers are exploring the use of shallower implants in a so-called multıpinned phase design [10]. At present there is a lot of activity in large-area [11] pixel arrays and the technology is improving rapidly, although relatively little attention is devoted to the $\mathrm{X}$-ray region at present

\section{$2.2 C C D$ readout}

During readout each row of stored charges is transferred to the serial register by means of the parallel electrode. The charge from each pixel is then transferred sequentially by the serial clock to an on-chip amplifier situated at the end of the serial register. Pixelto-pixel transfer takes approximately $6 \mu \mathrm{s}$. It is this combination of serial and parallel transfers that makes the CCD interesting for time-resolved applications. For example, if all pixels are masked except for the top row, the chip can be used as a streak camera to record a one-dimensional data set and to store hundreds of them in the masked area of the chip. The parallel row-to-row transfer time of $\sim 50 \mu \mathrm{s}$ offers a reasonably fast time resolution.

A detaled account of the readout electronics has been reported previously $[5,6]$. Fig. 3 summarizes the

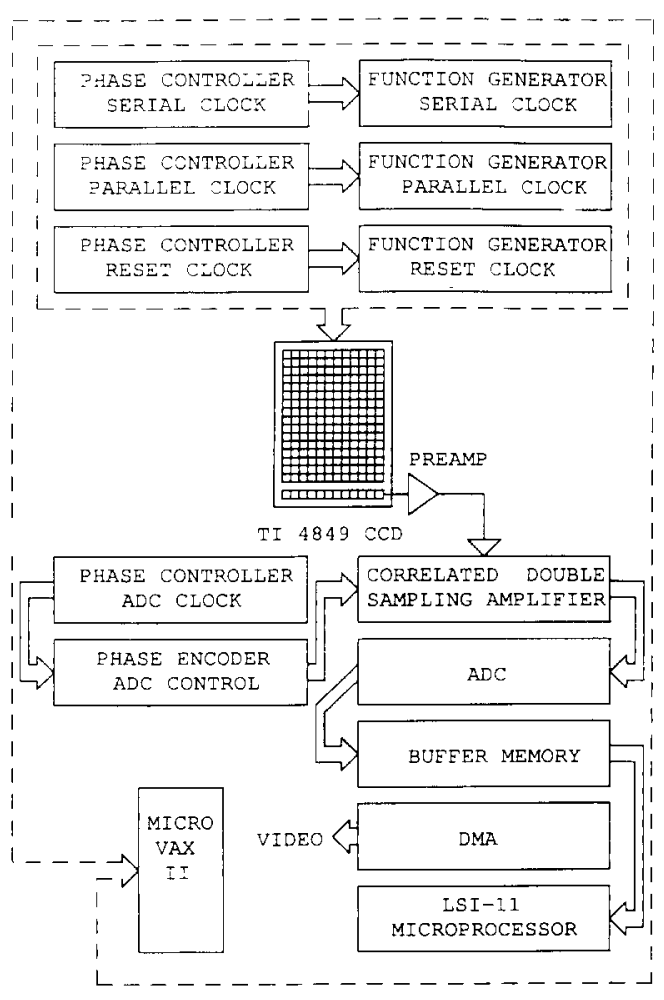

Fig 3. Block diagram of the CCD control and readout electronics. 

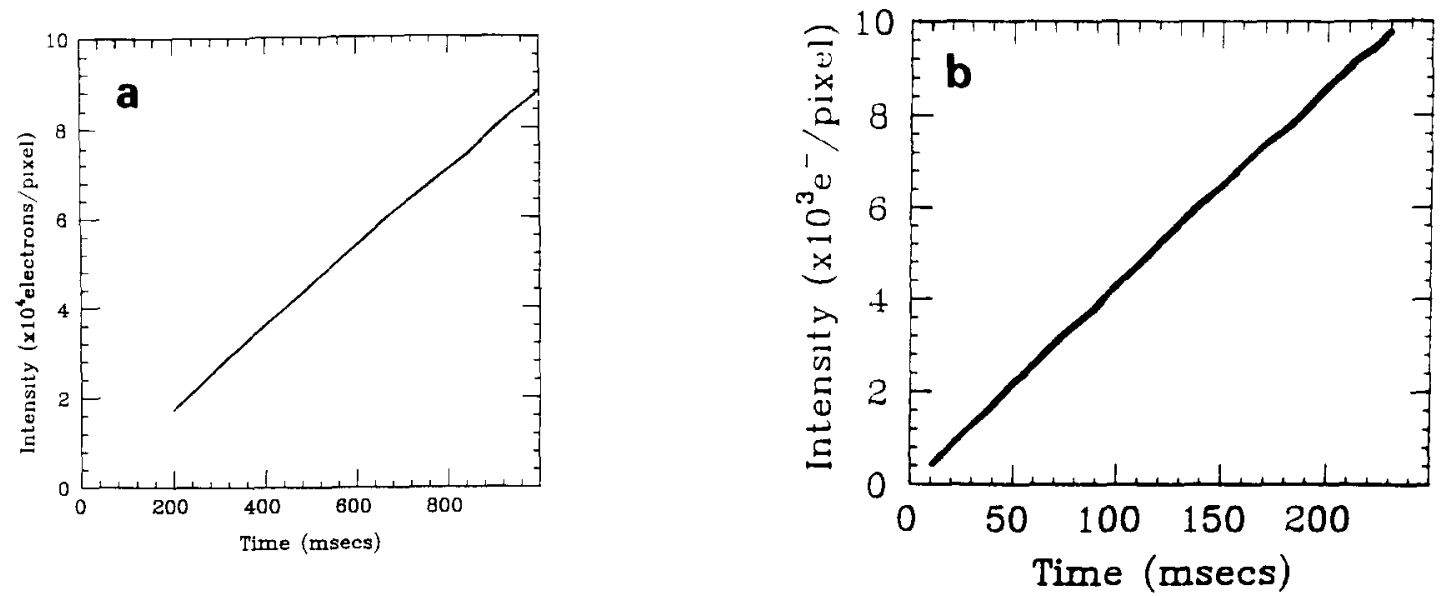

Fig. 4. (a) Detector linearity using optical Illumınation up to $45 \%$ of saturation. (b) Shows a blow-up of the linearity at low levels of 1llumination ( $\leq 5 \%$ of saturation). The horizontal axis refers to the exposure time.

main components of the CAMAC-based detector electronics, consisting of programmable waveform generators, analog-to-digital converter (ADC), buffer memory, clock synchronization modules, and local fast LSI-11based microprocessor control. Global control is handled by a Microvax II computer. All functions of the detector controls, readout, image display, and processing, are fully programmable; a diagnostic code has been included to isolate faults.

\section{Performance}

A series of standard performance tests [12] have been carried out on the CCD detector in order to determine its responsivity to both $\mathrm{X}$-rays $(6-12 \mathrm{keV})$

Table 1

Performance characteristics of the virtual phase CCD detector

\begin{tabular}{ll}
\hline CCD chip & TI4849 \\
Readout electronics & CAMAC \\
Frame size (pixels) & $584 \times 390$ \\
Readout noise & $20 \mathrm{e}^{-} / \mathrm{pixel}$ \\
Operatıng temperature & $-50^{\circ} \mathrm{C}$ \\
Quantum efficiency $(\lambda \approx 500 \mathrm{~nm})$ & $50 \%$ \\
Charge transfer efficiency & 0.999996 \\
Pixel well-depth & $200000 \mathrm{e}^{-}$ \\
Linearity & $<0.5 \%$ \\
Sensitivity (with phosphor screen & \\
$\quad$ and lens coupling) & $1 \mathrm{e}^{-} / \mathrm{X}_{\mathrm{ph}}$ \\
Spatial resolution & $2 \mathrm{pixels}(\approx 50 \mu \mathrm{m})$ \\
ADC resolution $(12$ bits) & $1 \mathrm{nn} 4 \times 10^{3}$ \\
Maximum dynamic range (to saturation) & $10^{4}$ \\
Readout time & $6 \mu \mathrm{s} / \mathrm{pixel}$ \\
Row parallel transfer time & $50 \mu \mathrm{s}$ \\
Full frame readout & $1 \mathrm{~s}$ \\
\hline
\end{tabular}

and optical photons $[5,6]$. Fig. 4 shows the results of a linearity test indicating better than $0.5 \%$ linearity over the typical operating range. Additional tests [6] show excellent uniformity ( $\pm 1 \%$ ), spatial resolution ( 2 pixels) and noise characteristics ( $<20 \mathrm{e}^{-} /$pixel). The operating characteristics are summarized in table 1 .

\section{Use as a position-sensitive detector}

The performance of the CCD system as a positionsensitive detector was evaluated on the DCI storage ring at LURE. Measurements were performed on a dispersive beamline in collaboration with Fontaine's group. Fig. 5 shows the experimental layout [13] with white bending-magnet radiation incident on a triangular Si(311) bent crystal which provided an energy-dispersed beam of bandwidth $\approx 500 \mathrm{eV}$. The sample is placed at the polychromatic focus point with the detector located on the Rowland circle.

One axis of the CCD (parallel to the pixel rows) is oriented accurately along the energy fan of the bent crystal so that the positional co-ordinate along this axis corresponds to a particular energy. In the perpendicular

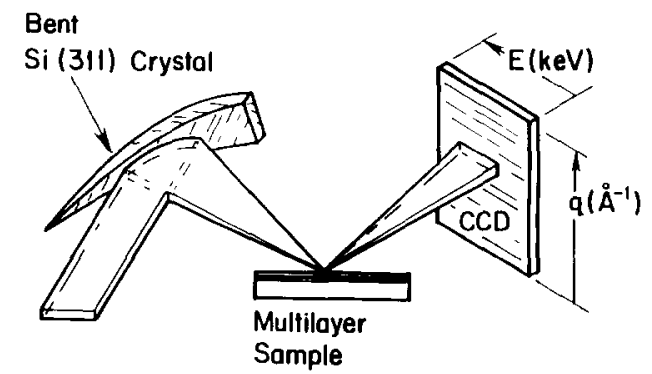

Fig. 5 Schematic X-ray optıcs for energy-dispersive measurements using a CCD detector. 


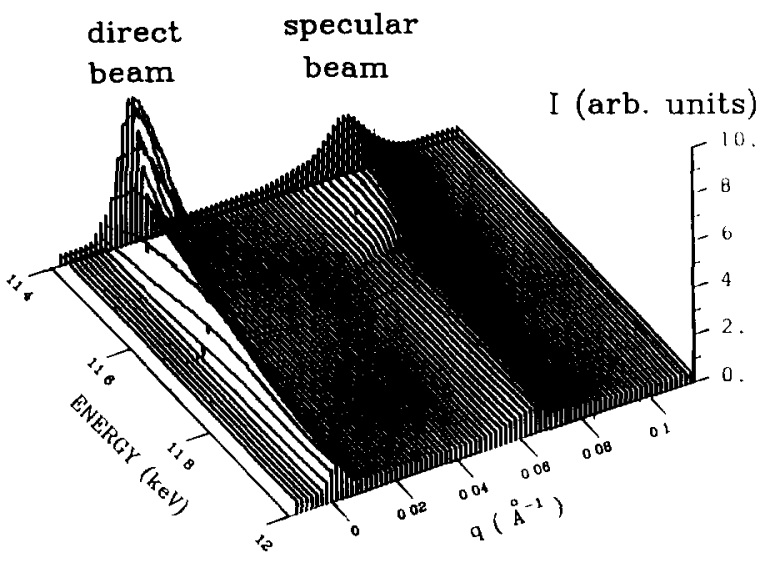

Fig. 6. Two-dimensional $\mathrm{CCD}$ readout of $\mathrm{Pt} / \mathrm{C}$ multilayer glancing-angle reflectivity. A part of the direct beam that misses the sample strikes the detector. The integration time for this data was $100 \mathrm{~ms}$.

direction, the detector registers the scattering angle (momentum transfer). In this way the detector can record spectroscopic data and scattering information simultaneously. An example of the readout from the CCD chip in such an experiment is shown in fig. 6 . The energy resolution obtained in this way is $-2 \mathrm{eV}$ and the wave-vector resolution, $1.5 \times 10^{-3} \AA^{-1}$. The specularly reflected beam from the surface of the $\mathrm{Pt} / \mathrm{C}$ multilayer sample clearly shows the $\mathrm{L}_{\mathrm{III}}$ absorption edge of $\mathrm{Pt}$ at $11.564 \mathrm{keV}$. Note that the data collection time is very short $(100 \mathrm{~ms})$ compared to conventional scanning techniques.

Reflectivity data extracted from this technique are plotted in fig. 7 for the $\mathrm{Pt} / \mathrm{C}$ multilayer close to its

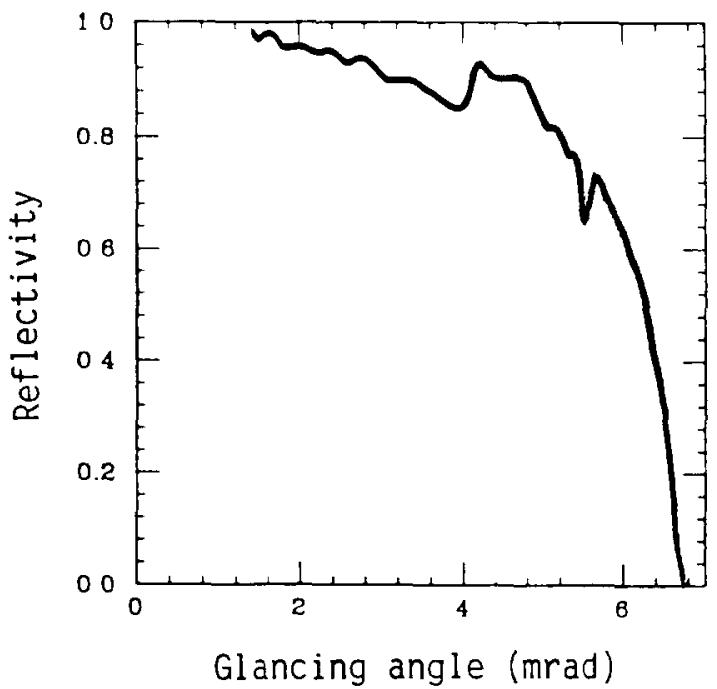

Fig. 7. Glancing-angle reflectivity curve for a $\mathrm{Pt} / \mathrm{C}$ multilayer measured at $11.56 \mathrm{keV}$ using the $\mathrm{CCD}$ detector. critical angle. The fine structure on this curve, such as the dip at a glancing angle of $\sim 4 \mathrm{mrad}$ is due to dynamical interference effects at the interface between the top Pt layer and the underlying carbon layer [14].

\subsection{Circular dichroism}

A second illustration of the power of position-sensitive detection coupled with dispersive optics is its recent application to the study of magnetic materials. Fig. 8 shows an energy dispersive arrangement similar to that in fig. 5 except that photons from just beneath the plane of the storage ring are selected; these have $\sim 80 \%$ circular polarization. The circularly polarized beam passes through the sample placed between the poles of an electromagnet which can switch the magnetization axis through $180^{\circ}$. The idea of this type of experiment is that there is a small difference between the near-edge absorption spectrum of the material when the spins of the magnetic d-electrons are parallel or antiparallel to the photoelectron polarization [15-17]:

$\Delta \mu_{\mathrm{K}} \sim\left|M_{1 \mathrm{~s}}\left(E_{\mathrm{x}}\right)\right|^{2} P_{\mathrm{e}} \Delta \rho(E)$,

where $P_{\mathrm{e}}$ is the photoelectron polarization $\left(\sim 10^{-2}\right)$, $M_{1 \mathrm{~s}}$ is the photon transition matrix element, and $\Delta \rho=$ $\rho \uparrow \uparrow-\rho \uparrow \downarrow$ is the difference in spin density between the majority and minority bands.

Magnetic absorption measurements such as those performed by Schütz et al. [17] at HASYLAB, using standard scanning monochromator methods, provide information about the spin density distribution of unoccupied states near the Fermi level. The information is somewhat complementary to spin-polarized XPS which normally probes the occupied states. Some of our preliminary measurements [18] (using dispersive optics and a one-dimensional diode array) are shown in fig. 9. The data in fig. $9 \mathrm{~b}$ refer to a superlattice of $\mathrm{Co}$ and $\mathrm{Au}$ grown by MBE with alternating layers of $10-\AA \mathrm{Co}$ and 16- $\AA \mathrm{Au}[19]$. The very thin layers of Co exhibit an easy axis of magnetization normal to plane of the film, i.e., parallel to the circularly polarized X-ray beam. Interestingly, the near-edge magnetic absorption spectrum of the superlattice appears to be enhanced relative to the bulk.

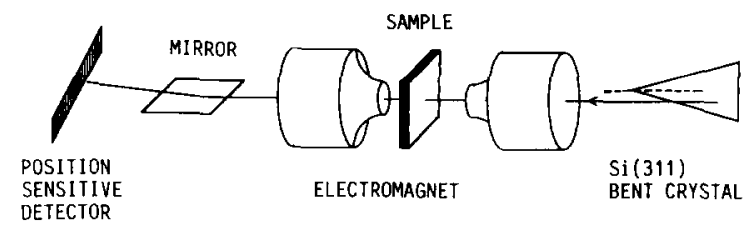

Fig. 8 Experimental arrangement for dispersive magnetic absorption measurements. The axis of the electromagnet is $4 \mathrm{~mm}$ below the plane of the ring [18]. 


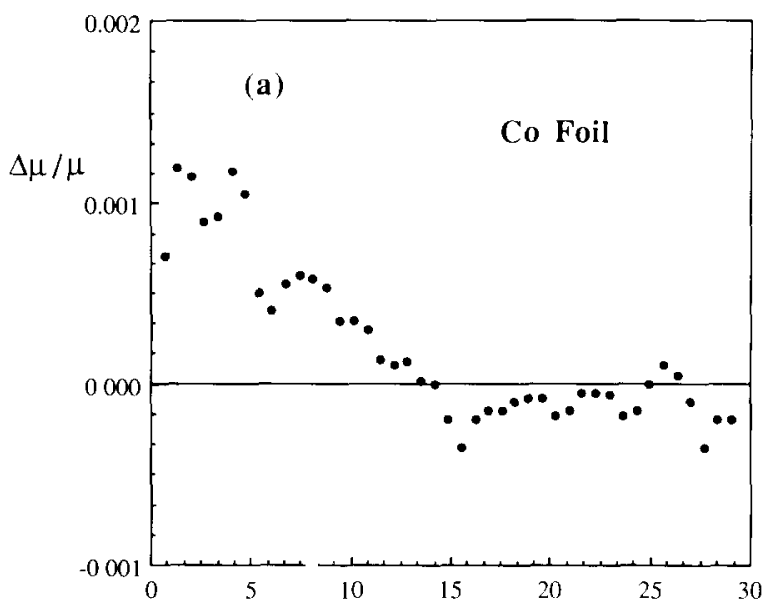

ENERGY (eV)

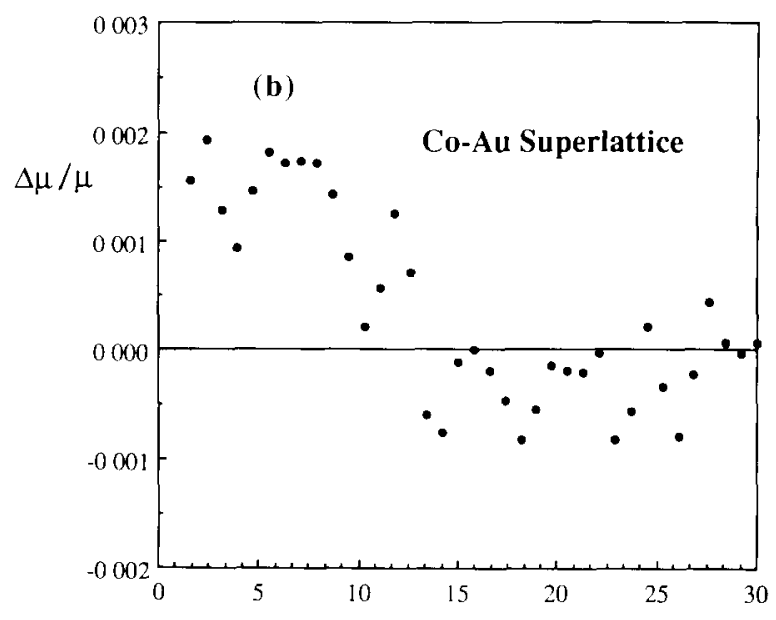

ENERGY (eV)

Fig. 9. Magnetic contribution of the near-edge absorption spectrum. The zero of the energy scale corresponds to the K-edge of Co at $7709 \mathrm{keV}$ (a) Cobalt foll; (b) Superlattice sample [18].

Here the advantage of the curved-crystal dispersive technique over a scanning monochromator is that the whole spectrum is collected simultaneously; alternate spectra with the magnetic field parallel and antiparallel to the X-ray beam are subtracted to obtain $\Delta \mu$. This method is very efficient; even so, the magnetic effect is quite weak and up to $2 \mathrm{~h}$ were required to obtain absorption spectra such as those shown in fig. 9. One problem is that the intensity falls off dramatically below the plane of the ring; the circularly polarized beam is only $\sim 10 \%$ of the linearly polarized beam intensity in the orbital plane. Helical undulators benng constructed for ESRF and APS will be very advantageous in this regard.

In this experiment only one polarization is recorded. With a two-dimensional CCD as a detector, the spectra for left and right circular polarizations could be recorded simultaneously, thus avoiding the use of a switching field and making more efficient use of the beam. An extension of this work includes the possibility to carry out magnetic EXAFS in which the magnetic short range order can be probed [20]. This would be particularly useful as a means to study the relationship between interface structure and magnetic anisotropy in ultrathin magnetic multilayers.

\section{Summary}

The development of fast high-resolution pixel-array detectors such as CCDs opens up a wide range of interesting new experimental techniques. While important hurdles still need to be overcome in their applications with high-brillance X-ray sources, we have demonstrated some very promising performance char- acteristics for this type of detector. The design of the $\mathrm{X}$-ray optics for the particular experiment will need to be considered carefully in conjunction with the detector system in order to exploit the capabilities of new highbrightness sources.

\section{Acknowledgements}

The work described in this artıcle is the result of a collaboration with E. Dartyge and A. Fontaine at LURE, R. Smither at Argonne, and F. Lamelas and B. Rodricks at University of Michigan; it is a pleasure to acknowledge the hospitality of LURE staff during the course of these experiments. The work was supported in part by NSF Grant DMR 8805156.

\section{References}

[1] See workshop report Time-Resolved Studies and Ultrafast Detectors, Argonne, ANL/APS-TM-2 (1988); S.M. Gruner, Science 238 (1987) 305.

[2] K.F. Ludwig, G.B. Stephenson, J.L Jordan-Sweet, J. Mainville, Y.S. Yang and M. Sutton, Phys. Rev. Lett. 61 (1988) 1859.

[3] R. Clarke and P. Hernandez, Phys. Rev. Lett. 62 (1989) 1768 ;

P. Hernandez, F. Lamelas, R. Clarke, P. Dimon, E.B. Sirota and S.K. Sinha, Phys. Rev. Lett. 59 (1987) 1220

[4] G. Tourllon, E. Dartyge, A. Fontaine and A Jucha, Phys. Rev. Lett. 57 (1986) 506.

[5] C.W. Akerlof, J.W Chapman, I. Gialas, W.A. Koska, D.F. Nitz, B.G. Rodricks and R.S. Tschırhart, Nucl Instr. and Meth. A260 (1987) 80. 
[6] B.G. Rodricks, R Clarke, R. Smither and A. Fontaine. Rev Sci. Instr., 60 (1989) 2586.

[7] Note that the TI 4849 is no longer available and has been superseded by another chip, TI 215.

[8] J Hynecek, IEEE Trans. Electron Dev. ED-28 (1981) 483.

[9] R D McGrath, IEEE Trans Nucl Sci. NS-28 (1981) 4028.

[10] J. Janesick, T. Ellott, M. Blouke and B. Corrie, SPIE Optical Sensors and Electronic Photography 1071 (1989) p 153

[11] See, for example, R.A. Bredthauer, SPIE New Methods in Microscopy and Low Light Imaging, 1161 (1989) p. 61.

[12] S.M Gruner and J.R. Milch, Trans ACA 18 (1982) 149.

[13] E Dartyge. C Depautex, J.M. Dubuisson, A. Fontaine, A. Jucha, P. Leboucher and G. Tourillon, Nucl. Instr. and Meth. A246 (1986) 452.
[14] B Rodricks, F. Lamelas, D. Medjahed, W Dos Passos, R. Smither, E. Ziegler, A. Fontaine and R. Clarke. Mater Res. Soc. Symp. Proc 143 (1989) 209.

[15] E. Keller and E.A. Stern, in: EXAFS and Near Edge Structure III (Sprınger, Berlın, 1984) p. 507.

[16] G. Schütz and R. Wienke, Proc. Int. Conf. on Nuclear Methods in Magnetısm. Munich, 1989, in press.

[17] G. Schütz, W Wagner, W Wilhelm, P. Kıenle, R. Zeller, R. Frahm and G. Materlı, Phys. Rev. Lett. 58 (1987) 737.

[18] F Lamelas, E Dartyge, A Fontaine and R. Clarke, to be published.

[19] C.H. Lee, Huı He, F. Lamelas, W. Vavra, C. Uher and R Clarke. Phys. Rev. Lett. 62 (1989) 653.

[20] G. Schütz, R Frahm. P. Mautner, R. Wienke, W. Wagner, W Wilhelm and P. Kienle. Phys. Rev. Lett 62 (1989) 2620. 